\title{
Speed of reaction-transport processes
}

\author{
Vicenç Méndez ${ }^{1}$ and Joaquim Fort ${ }^{2}$ \\ ${ }^{1}$ Facultat de Ciències de la Salut, Universitat Internacional de Catalunya, c/Gomera s/n, 08190 Sant Cugat del Vallès, \\ Catalonia, Spain \\ ${ }^{2}$ Departament de Física, Universitat de Girona, Campus de Montilivi, 17071 Girona, Catalonia, Spain
}

(Received 12 December 2000; published 14 June 2001)

\begin{abstract}
We present an approach to determining the speed of wave-front solutions to reaction-transport processes. This method is more accurate than previous ones. This is explicitly shown for several cases of practical interest: (i) the anomalous diffusion reaction, (ii) reaction diffusion in an advective field, and (iii) time-delayed reaction diffusion. There is good agreement with the results of numerical simulations.
\end{abstract}

DOI: 10.1103/PhysRevE.64.0111XX PACS number(s): 05.40. $-\mathrm{a}, 47.70 .-\mathrm{n}, 87.10 .+\mathrm{e}, 05.60 . \mathrm{Cd}$

Many natural phenomena, such as the propagation of combustion waves, solidification, superconducting fronts, and the dispersal of biological populations, are driven by an interplay of transport and reactive processes. For the sake of clarity, let us begin with a specific example. Consider the equation

$$
\partial_{t} \rho=\partial_{x x}\left(\rho^{m}\right)+f(\rho),
$$

where $\rho$ is a time-dependent field (particle number density, temperature, etc.), $f(\rho)$ is a nonlinear function, and $m \geqslant 1$. The case $m=1$ corresponds to Fickian diffusion, whereas $m>1$ arises, e.g., from a density-dependent diffusion coefficient or a temperature-dependent thermal conductivity. The first and second terms on the right-hand side (RHS) in Eq. (1) are related, respectively, to the transport and source processes of mass, energy, or electric charge. Such equations admit the so-called front solutions, which describe the shape and evolution of the field from its unstable (initial) state to the stable (final) state. This shape may correspond to the population profile of a biological population, the temperature profile in a flame, etc. Usually $\rho(x, t)$ is defined such that $\rho=0$ and $\rho=1$ correspond to the unstable and stable states, respectively. In general it is not possible to find such solutions explicitly in analytical form. However, a problem of utmost importance is to determine their asymptotic velocity at large times in order to compare this prediction to values from experiments where one measures the speed of propagation of biological populations [1], combustion fronts [2], etc.

Several methods exist for the analytical calculation of the speed $c$ of front solutions to reaction-diffusion equations, such as Eq. (1). Dee and Langer [3] proposed a linearization approach, which works for simple equations with $m=1$ and $f^{\prime}(0) \neq 0$. Paquette and co-workers [4] used a renormalization group (RG) method which allows us to find approximate results for the speed when a perturbation in introduced. This requires us to know the exact solution for the unperturbed equation, and works for small perturbations only. Benguria and Depassier (BD) discovered an alternative approach [5] which is based on a variational method. Their procedure requires using trial functions which are a priori unknown. By judicious use of such trial functions, one may find accurate lower and upper bounds for the speed. Only if the lower and upper bounds coincide can the value of $c$ be determined without any uncertainty [6]. In practice, the problem arises that sometimes one is not able to find out suitable trial functions such that the lower and upper bounds coincide [7]. Then, the speed cannot be determined precisely.

We will here present a method that relies on some approximations but agrees rather well to numerical simulations and improves the results obtained by means of previous approaches in several relevant applications. It has the attractive features that it is not necessary to know the exact solution for an unperturbed equation (in contrast to the RG approach) nor is it necessary to use a priori unknown trial functions (in contrast to the BD approach). Our method is based on a modification to the BD approach. Again for the sake of clarity, we shall introduce it for some relevant examples so as to avoid using an abstract notation.

\section{ANOMALOUS DIFFUSION REACTION}

Consider first Eq. (1). We look for solutions of the form $\rho=\rho(x-c t)$, where $c>0$ is the speed of a front moving to the right. Defining $z=x-c t$, Eq. (1) becomes an ordinary differential equation, namely,

$$
\left(\rho^{m}\right)_{z z}+c \rho_{z}+f(\rho)=0,
$$

where $f(0)=f(1)=0$ and $\lim _{z \rightarrow-\infty} \rho=1, \lim _{z \rightarrow \infty} \rho=0$. Benguria and Depassier [5] have shown that the speed $c$ follows from a variational principle and is given by

$$
c=\max _{g}\left[2 \frac{\int_{0}^{1} \sqrt{m \rho^{m-1} f g h} d \rho}{\int_{0}^{1} g d \rho}\right],
$$

where $g>0$ is a function such that $h \equiv-d g / d \rho>0[5,8]$, and it satisfies the equation

$$
\frac{1}{m} \rho^{m-1} f \frac{g}{p}=h p,
$$

with $p \equiv-\rho^{m-1} \rho_{z}$. Since the front moves to the right and $\rho=1$ is the stable state, we have $p>0$.

The asymptotic speed of the front for sufficiently localized initial conditions [9] may be determined in the limit $t$ $\rightarrow \infty$. In this limit one has $\rho \rightarrow 1$ for $z \rightarrow-\infty$, and $\rho$ is a slowly varying function of $z$. Therefore one has $\left(\rho^{m}\right)_{z z}$ 
$\ll \rho_{z}$, and from Eq. (2) we have $c \rho_{z}+f(\rho) \simeq 0$ and $p$ $\simeq-\rho_{z} \simeq f(\rho) / c$. This is an approximate relationship, so that we may use $p=f(\rho) / \alpha>0$, where $\alpha$ is a positive constant to be determined and the trial function $g$ is, by virtue of Eq. (4),

$$
g(\rho)=\exp \left[-\frac{\alpha^{2}}{m} \int \frac{\rho^{m-1}}{f(\rho)} d \rho\right] .
$$

The maximum in Eq. (3) must be taken over $\alpha$ and the speed may be calculated from

$$
c \simeq \max _{\alpha \in \mathcal{D}}\left(2 \alpha \frac{\int_{0}^{1} \rho^{m-1} g d \rho}{\int_{0}^{1} g d \rho}\right),
$$

where $\mathcal{D}$ is the set of positive values of $\alpha$ such that the integrals in Eq. (6) exist.

As a simple illustration, consider the well-known Fisher equation $\partial_{t} \rho=\partial_{x x} \rho+\rho(1-\rho)$. In this case $c$ $\simeq \max _{\alpha \in \mathcal{D}}(2 \alpha)$ where $\alpha$ is such that $\int_{0}^{1} g d \rho$ exists. From Eq. (5), taking $m=1$ and $f=\rho(1-\rho)$ one finds $g=\rho^{-\alpha^{2}}(1$ $-\rho)^{\alpha^{2}}$, thus the integral $\int_{0}^{1} g d \rho$ exists if $\alpha<1$ [10], so in this case $\mathcal{D}=(0,1)$ and we recover the well-known result $c$ $\simeq \max _{0<\alpha<1}(2 \alpha)=2$, where the maximum is attained for $\alpha$ $=1$.

Now consider the case $m>1$. Then, Eq. (1) without the last term corresponds to anomalous diffusion, which arises from Tsallis statistics [11]. When the last term in Eq. (1), which corresponds to a source or reactive process, is included, Eq. (1) is useful in genetics and population dynamics [12-14], as well as in many combustion fronts [15-17]. The exact speed of such fronts is known only for the case $m$ $=2$ and a logistic growth function, namely $f=\rho(1-\rho)$, for which $\rho=\left(1+e^{z / 2}\right) \theta(-z)$ with $c=1$ [18]. In our approach, we may recover this result but the explicit solution for $\rho(z)$ is not needed: from Eq. (5) one finds $g=(1-\rho)^{\alpha^{2} / 2}$, thus $\int_{0}^{1} g d \rho=\left(1+\alpha^{2} / 2\right)^{-1} \quad$ and $\quad \int_{0}^{1} g \rho d \rho=\left(2+\alpha^{2} / 2\right)^{-1}(1$ $\left.+\alpha^{2} / 2\right)^{-1}$. Therefore $\mathcal{D}=(0, \infty)$ and $c \simeq \max _{\alpha \in(0, \infty)}[2 \alpha /(2$ $\left.\left.+\alpha^{2} / 2\right)\right]=1$, where the maximum is attained for $\alpha=2$. For other values of $m$, the integrals involved in Eq. (6) must be computed numerically. For instance, for the case $m=3 / 2$ we obtain from Eq. (5) $g=(1-\sqrt{\rho})^{2 \alpha^{2} / 3}(1+\sqrt{\rho})^{-2 \alpha^{2} / 3}$, the maximum in Eq. (6) is attained for $\alpha=1.6$ and $c \simeq 1.198$. In Fig. 1 we present our results for the speed $c$ (full line) and compare them to those obtained previously by other methods and the results of numerical simulations [19]. From Fig. 1 it is seen that our method is extremely precise, and improves the results of the $\mathrm{RG}[4,20]$ and $\mathrm{BD}[5,8]$ approaches. We now turn to Fickian diffusion $(m=1)$ and show how our approach may be used to obtain better results than those derived previously.

\section{REACTION DIFFUSION UNDER ADVECTION}

In many physical and biological applications, one deals with a reacting species which has a mean speed in the labo-

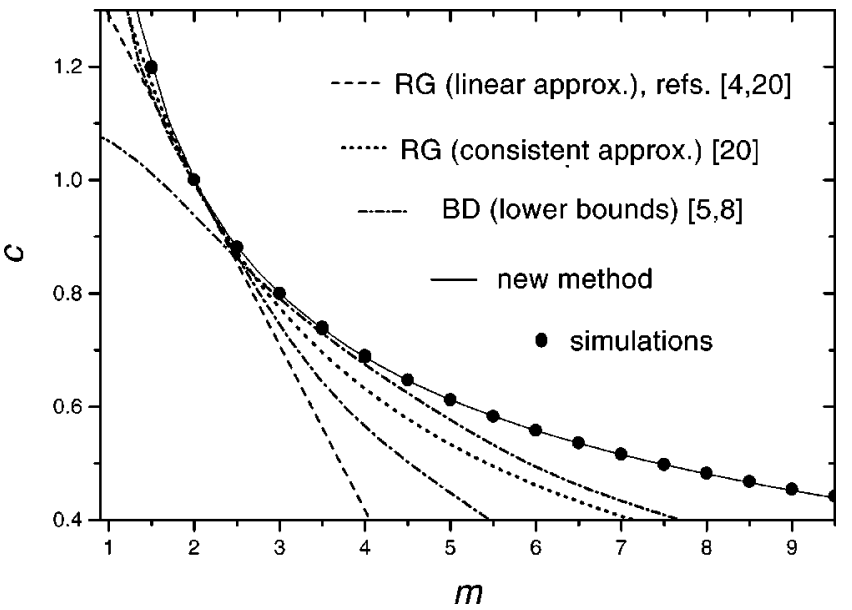

FIG. 1. Predictions for the speed of wave-front solutions to Eq. (1) for $f(\rho)=\rho(1-\rho)$, according to the RG approach $c \simeq 1-7(m$ $-2) / 24$, dashed curve, and $c \simeq[1+7(m-2) / 24]^{-1}$, dotted curve $[4,20]$ and to our method presented here (full curve). The dasheddotted curves are lower bounds predicted from the BD approach $[5,8]$, and the black circles are the results of numerical simulations.

ratory frame. In this context, equations of the following form have been considered:

$$
\partial_{t} \rho+g(\rho) \partial_{x} \rho=\partial_{x x} \rho+f(\rho),
$$

where the second term, which contains the field gradient $\partial_{x} \rho$, is used to model the effect of convection [21]. Such terms have been applied to the evolution of bacterial colonies under convection [22] and to the transport of micro-organisms in ground water [23]. They have also been proposed in the study of turbulent flames [24], in combustion under convective forcing [25], etc. However, up to now very scarce analytical results have been derived. With the purpose of illustrating the application of our method, let us consider a reaction-diffusion-advection equation of the form of Eq. (7) which has been often dealt with in the literature $[21,26,27]$,

$$
\partial_{t} \rho+\mu \rho \partial_{x} \rho=\partial_{x x} \rho+f(\rho),
$$

for which Murray was able to derive a lower bound for the propagation speed of wavefronts [21]. For logistic growth it reads

$$
c \geqslant \begin{cases}2 & \text { if } \mu<2, \\ \frac{2}{\mu}+\frac{\mu}{2} & \text { if } \mu \geqslant 2 .\end{cases}
$$

On the other hand, recently an upper bound for the same fronts has been found by means of the BD approach [27],

$$
c \leqslant \begin{cases}2 & \text { if } \mu<2, \\ \mu & \text { if } \mu \geqslant 2 .\end{cases}
$$

Comparison of Eqs. (9) and (10) shows that the wavefront speed can be predicted precisely only in the case of small advective effects $(\mu<2)$. This may also be seen in Fig. 2, where the prediction of the $\mathrm{RG}$ approach is also shown [28]. 




FIG. 2. Reaction diffusion under an advection field. Previously, only lower [21] and upper [27] bounds had been derived. Our method makes a precise prediction, which is in good agreement with simulations.

Let us show that our method allows us to determine the speed without any ambiguity under arbitrarily strong advection.

Introducing $z=x-c t$, Eq. (8) becomes

$$
\rho_{z z}+(c-\mu \rho) \rho_{z}+f(\rho)=0 .
$$

Defining $p=-\rho_{z}$, we have $p=0$ at $\rho=0,1$. Then, multiplying Eq. (11) by $g / \rho$ (where $g>0$ is any function such that $d g(d \rho<0)$, and integrating with respect to $\rho$, we may apply that integration by parts yields $\int_{0}^{1} g d p=\int_{0}^{1} p h d \rho$, where $h$ $\equiv-d g / d \rho$. Using Schwarz's inequality $h p+f g / p \geqslant 2 \sqrt{f g h}$ [29] it is easy to find that [27]

$$
c=\max _{g}\left(2 \frac{\int_{0}^{1} \sqrt{f g h} d \rho}{\int_{0}^{1} g d \rho}+\mu \frac{\int_{0}^{1} g \rho d \rho}{\int_{0}^{1} g d \rho}\right),
$$

where the maximum is attained for $g$ such that it satisfies $h p=f g / p$ [30] or $\sqrt{f g h}=f g / p$. Considering Eq. (11) in the limit $\rho \rightarrow 1$ and proceeding as in the previous example, we see that we may again take $p \simeq f(\rho) / \alpha$. Thus

$$
c \simeq \max _{\alpha \in \mathcal{D}}\left(2 \alpha+\mu \frac{\int_{0}^{1} g \rho d \rho}{\int_{0}^{1} g d \rho}\right),
$$

where $g$ may be calculated from the differential equation $d g / g=-\alpha^{2} d \rho / f$ which yields, after integration, $g$ $=\exp \left(-\alpha^{2} \int d \rho / f\right)$. For the logistic growth $f=\rho(1-\rho)$ one finds $g=\rho^{-\alpha^{2}}(1-\rho)^{\alpha^{2}}$ and therefore $c=\max _{0<\alpha<1}[2 \alpha$ $\left.+\mu\left(1-\alpha^{2}\right) / 2\right]=2 / \mu+\mu / 2$, which is attained for $\alpha=\mu / 2<1$, that is, for $\mu>2$. For $\mu<2$, however, the maximum occurs as $\alpha \rightarrow 1$ and yields $c=2$. Therefore we get an analytic result for the speed,

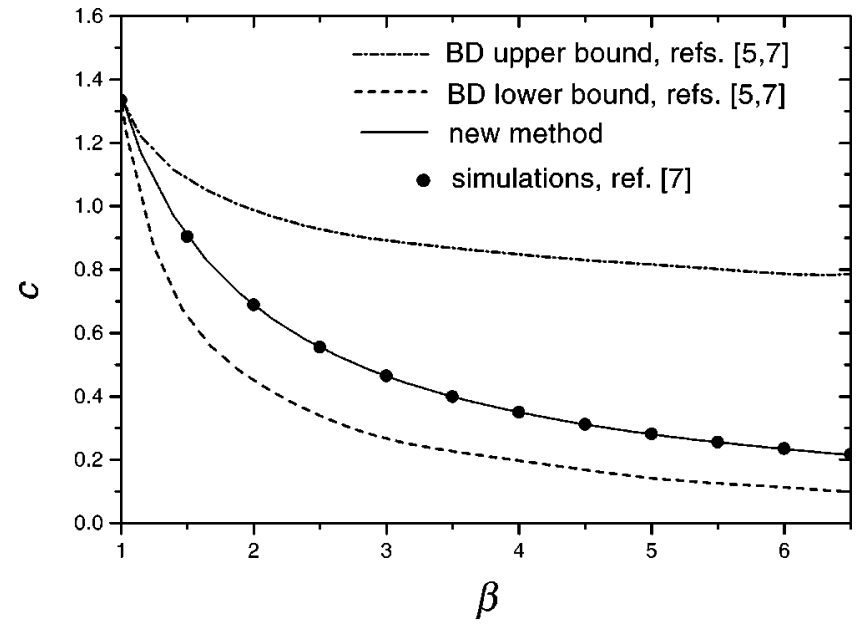

FIG. 3. Nonlinear reaction under time-delayed diffusion. The upper and lower bounds [7] from the BD approach [5] are improved by our method. In this way, the speed of wave fronts can be predicted precisely.

$$
c \simeq\left\{\begin{array}{l}
2 \quad \text { if } \mu<2 \\
\frac{2}{\mu}+\frac{\mu}{2} \quad \text { if } \mu \geqslant 2 .
\end{array}\right.
$$

In Fig. 2, this result is seen to agree very well with those obtained by means of numerical simulations of Eq. (7) with $f=\rho(1-\rho)$. We note that this eliminates the ambiguity of the lower and upper bounds [Eqs. (9) and (10)] previously derived by other methods [21,27], and it also improves the accuracy of the RG approach.

\section{TIME-DELAYED REACTION DIFFUSION}

Finally let us illustrate our method for a nonlogistic source function and higher-order time derivatives. In many applications such as population dynamics [1,31] and nerve conduction [7], the transport of mass, heat, or electric charge is time delayed and an equation of the following form arises:

$$
a \partial_{t t} \rho+\partial_{t} \rho=\partial_{x x} \rho+f(\rho)+a f^{\prime}(\rho) \partial_{t} \rho .
$$

An interesting source function is $f=\rho^{\beta}(1-\rho)$, which has been applied in forest fire research [32,7] and microbiology [33]. However, in this case only lower and upper bounds for the speed of front solutions to Eq. (13) have been determined previously [7]. Let us show how our method makes it again possible to find an accurate value for the speed. In Ref. [7] we have shown from the BD method [5] that the speed of the fronts described by Eq. (13) satisfies the variational principle

$$
\frac{c}{\sqrt{1-a c^{2}}}=\max _{g}\left[2 \frac{\int_{0}^{1} \sqrt{f g h} d \rho}{\int_{0}^{1} g\left(1-a f^{\prime}\right) d \rho}\right],
$$

where $h \equiv-d g / d \rho, f^{\prime} \equiv d f / d \rho$, and $p \equiv-\rho_{z}$, and the function $g$ for which the parentheses in the RHS is maximum 
satisfies $\left(1-a c^{2}\right) h p=f g / p$. In the limit $\rho \rightarrow 1$ one has, since $\rho_{z z} \ll \rho_{z}$ as in previous cases, $-c\left(1-a f^{\prime}\right) p+f(\rho)$ $\simeq 0$ and we take $p=f \alpha^{-1}\left(1-a f^{\prime}\right)^{-1}$. For the source function $f=\rho^{\beta}(1-\rho)$, in the limit $\rho \rightarrow 1$ one may approximate $f \simeq \rho(1-\rho)$ and, proceeding as in Secs. I and II, we find that we may now use $p \simeq \rho(1-\rho) \alpha^{-1}\left(1-a f^{\prime}\right)^{-1}$.

Finally, the speed may be determined from

$$
c \simeq \max _{\alpha \in \mathcal{D}}\left\{2 \alpha \frac{\int_{0}^{1} g \rho^{\beta-1}\left[1-a f^{\prime}\right] d \rho}{\int_{0}^{1} g\left[1-a f^{\prime}\right] d \rho}\right\},
$$

where $g=\exp \left\{-\alpha^{2} \int \rho^{\beta-2}(1-\rho)^{-1}\left[1-a f^{\prime}\right]^{2} d \rho /\left(1-a c^{2}\right)\right\}, \quad f^{\prime}$ $=d f / d \rho=\beta \rho^{\beta-1}-(1+\beta) \rho^{\beta}$, and $\mathcal{D}$ is the set of values for $\alpha$ such that the integrals exist.

For illustration purposes, consider, e.g., the case $a=1 / 2$. We perform the integrals in $g$ and Eq. (14) numerically, as in Sec. I above, and in Fig. 3 we see that the new results agree with the direct simulations [7] of Eq. (13) with $f=\rho^{\beta}(1$ $-\rho$ ). Whereas the RG approach cannot be applied since no exact solution for Eq. (13) is known for $a=0$ and arbitrary $\beta$ or vice versa, the $\mathrm{BD}$ predictions are clearly improved also in this case.

Our approach relies on the $\mathrm{BD}$ variational principle, for which we provide a specific trial function $g$ which leads to very accurate results. We would like to stress that the usefulness of the method here reported is not restricted to any condition on the source function $f(\rho)$ nor to specific differential operators in the reaction-transport equation. Figures 1-3 show that the method substantially improves the results obtained previously from alternative approaches [3-5]. Details and further applications will be presented elsewhere [34].

\section{ACKNOWLEDGMENTS}

We are grateful to D. Jou for pointing out the relevance of anomalous diffusion. Computing equipment was funded in part by the CICYT of the Ministry of Science and Technology under Grant Nos. BFM2000-0351 (V.M. and J.F.) and No. REN2000-1621 CLI (J.F.).
[1] J. Fort and V. Méndez, Phys. Rev. Lett. 82, 867 (1999); Phys. Rev. E 60, 5894 (1999)

[2] J. Fort, T. Pujol, and A. S. Cukrowski, J. Phys. A 33, 6953 (2000).

[3] G. Dee and J. S. Langer, Phys. Rev. Lett. 50, 383 (1983).

[4] G. C. Paquette, L-Y. Chen, N. Goldenfeld and Y. Oono, Phys. Rev. Lett. 72, 76 (1994).

[5] R. Benguria and M. C. Depassier, Phys. Rev. Lett. 73, 2272 (1994).

[6] R. Benguria and M. C. Depassier, Phys. Rev. E 57, 6493 (1998).

[7] V. Méndez, J. Fort, and J. Farjas, Phys. Rev. E 60, 5231 (1999).

[8] R. Benguria and M. C. Depassier, Phys. Rev. E 52, 3258 (1995)

[9] D. G. Aronson and H. F. Weinberger, Adv. Math. 30, 33 (1978).

[10] S. Gradshteyn and I. M. Ryzhik, Table of Integrals, Series and Products (Academic, San Diego, 1994), formula 3.192-1.

[11] M. Bologna, C. Tsallis, and P. Grigolini, Phys. Rev. E 62, 2213 (2000); D. H. Zanette and P. A. Alemany, Phys. Rev. Lett. 75, 366 (1995).

[12] W. S. C. Gurney and R. M. Nisbert, J. Theor. Biol. 52, 441 (1975).

[13] M. E. Gurtin and R. C. MacCamy, Math. Biosci. 33, 35 (1977).

[14] S. Harris, Phys. Rev. E 62, 4032 (2000).

[15] L. A. Vulis, Thermal Regimes of Combustion (McGraw-Hill, New York, 1961).

[16] Ya. B. Zeldovich, G. I. Barenblatt, V. B. Librovich, and G. M. Makhviladze, The Mathematical Theory of Combustion and Explosions (Consultants Bureau, New York, 1985).

[17] J. Göttgens, F. Mauss, and N. Petters, in 24th Symposum (International) on Combustion (The Combustion Institute, Pittsburgh, 1992), pp. 129-135.
[18] W. I. Newman, J. Theor. Biol. 85, 325 (1980).

[19] The results of our simulations agree with those by Newman [18] for the cases he considered $(m=2,3)$. It is very interesting to note that, according to Eq. (3), the equation $\partial_{t} \rho=\partial_{x x} \rho$ $+m \rho^{m-1} f(\rho)$ has the same speed of wave fronts as Eq. (1), although the shape of the fronts will be different in general. This gives an additional method to check the speed of fronts by means of numerical simulations.

[20] L-Y Chen, N. Goldenfeld, and Y. Oono, Phys. Rev. E 49, 4502 (1994).

[21] J. D. Murray, Mathematical Biology (Springer-Verlag, Berlin, 1993).

[22] T. Neicu et al., Phys. Rev. E 62, 1059 (2000).

[23] Y. Tan et al., Water Resour. Res. 30, 3243 (1994).

[24] A. F. Ghoniem, M. C. Soteriou, and O. M. Knio, in 24th Symposium (International) on Combustion, Ref. [17], pp. 223230.

[25] N. Provatas et al., Phys. Rev. E 51, 4232 (1995).

[26] K. Lika and T.G. Hallam, J. Math. Biol. 38, 346 (1999).

[27] R. Benguria, M. C. Depassier, and V. Méndez (unpublished).

[28] The RG result follows from Eq. (9) in [4] or Eq. (2.10) in [20] by noting that for Eq. (11) the perturbation about $\mu=2$ is $\delta F=(\mu-2) \rho_{0} d \rho_{0} / d z$, and using the weight function $e^{z}$ and $\rho_{0}=\rho_{\mu=2}=\left(1+e^{z}\right)^{-1}$. This yields $c \simeq 2-11(\mu-2) / 560$. Note that we can use this $\rho_{0}$ because the lower and upper bounds (9) and (10) coincide, so it has the selected speed (we stress that this does not happen for all exact solutions [21]).

[29] For any positive numbers $r$ and $s$, it follows directly from $(r$ $-s)^{2} \geqslant 0$ that $(r+s) \geqslant 2 \sqrt{r s}$ (Schwartz's inequality).

[30] This follows immediately from the fact that the equalities in [29] hold for $r=s$.

[31] V. Méndez and J. Camacho, Phys. Rev. E 55, 6476 (1997).

[32] V. Méndez and J. E. Llebot, Phys. Rev. E 56, 6557 (1997).

[33] K. M. Vereecken, E. J. Dens, and J. F. Van Impe, J. Theor. Biol. 205, 53 (2000).

[34] V. Méndez and J. Fort (unpublished). 